Nathalie Marceau

Université de Sherbrooke (Canada)

\section{Autorégulation de l'apprentissage professionnel et pratiques d'enseignement: intersections à considérer pour le développement professionnel des enseignants}

\author{
Self-Regulated Learning and Teaching Practices: \\ Intersections to Consider for Teacher Professional Development
}

doi: $10.18162 / f \mathrm{fp} .2021 .560$

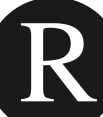
ésumé

Cette recherche décrit l'autorégulation de I'apprentissage d'enseignants en exercice au cours du développement d'une compétence professionnelle. Une recherche descriptive, réalisée sous la forme d'une étude de cas multiples, a été effectuée par le biais d'entrevues, de séances d'observation, d'entretiens d'explicitation et d'artefacts. Une analyse qualitative des données par théorisation ancrée a été effectuée avec

NVivo. La recherche a permis de dégager quatre intersections entre les phases des pratiques d'enseignement et de l'autorégulation de l'apprentissage qui offrent des avenues à considérer pour le développement professionnel.

Mots-clés

Autorégulation de l'apprentissage, développement professionnel, enseignants en exercice, pratiques d'enseignement.

Abstract This research describes the self-regulated learning of in-service teachers during the development of a professional competence. A descriptive multiple case study was conducted through interviews, observation sessions, and artifacts. A qualitative analysis of the data by grounded theory was performed with NVivo. This research has identified

four intersections between phases of teaching practice and of self-regulated learning that offer avenues to consider for professional development.

Keywords

Self-regulated learning, professional development, teaching practice, in-service teachers.

\section{Introduction}

Le ministère de l'Éducation du Québec (MEQ) souligne que «la formation continue est incontournable, la formation initiale n'en constituant que le premier jalon» (Gouvernement du Québec, 2020, p. 84). Les enseignants devant veiller à leurs apprentissages professionnels, il semble pertinent de s'intéresser à la régulation qu'ils en font (Gouvernement du Québec, 2020).

Cet article introduit la problématique et le cadre théorique avant de décrire le processus itératif ayant permis de constater quatre intersections entre les phases des pratiques d'enseignement $(\mathrm{PE})$ et celles de l'autorégulation de l'apprentissage (ARA). La présentation et la discussion des résultats illustrent le tout et permettent de soulever quelques pistes pour soutenir le développement professionnel des enseignants en exercice.

\section{Problématique}

Cette recherche s'inscrit dans le contexte professionnel du développement de la compétence «adapter ses interventions aux besoins et aux caractéristiques des élèves présentant des difficultés d'apprentissage, d'adaptation ou un handicap» (Gouvernement du Québec, 2001, p. 103) ${ }^{1}$. Le MEQ s'attend également à ce que le personnel enseignant applique les politiques et les normes touchant ces élèves (Gouvernement du Québec, 1999, 2008).

Or, la formation initiale des enseignants soulève plusieurs problèmes. Un changement de paradigme a été apporté aux programmes universitaires de formation initiale à l'enseignement en 1994 et en 2001 (Lenoir, 2010). Un écart existe entre la nature de la formation et la perception des futurs enseignants quant à leur niveau de 
préparation «à la réalité de la classe» (Goyette et Martineau, 2018). Un nombre limité de crédits est consacré aux élèves en difficulté dans ces programmes. D'ailleurs, le récent référentiel de compétences de la profession enseignante souligne que la formation initiale «ne permet pas d'approfondir toutes les compétences professionnelles»(Gouvernement du Québec, 2020, p. 84).

Pourtant, les besoins de formation continue pour développer cette compétence sont importants, comme le soulignent de nombreux acteurs de l'éducation : universités, enseignants en exercice, gouvernement, chercheurs et syndicats (Fédération des syndicats de l'enseignement (FSE), 2014; Gaudreau, Royer, Frenette, Beaumont et Flanagan, 2013; Gouvernement du Québec, 2010).

De plus, les enseignants doivent s'engager dans une démarche individuelle et collective de développement professionnel (Gouvernement du Québec, 2020). Les recherches sur le développement professionnel soulignent qu'il est favorisé lorsqu'il s'effectue sur une durée permettant l'enracinement des apprentissages (Capps, Crawford et Constas, 2012; Waitoller et Artiles, 2013). L'aspect social joue également un rôle important. Ainsi, la présence d'un soutien extérieur en dehors de la formation (Capps et al., 2012) de même que la collaboration avec les collègues (Waitoller et Artiles, 2013) sont à considérer. L'utilisation de situations authentiques, l'élaboration d'activités d'enseignement pendant et à la suite de la formation et la discussion sur le transfert de l'apprentissage vers la pratique sont également mentionnées (Bakkenes, Vermunt et Wubbels, 2010; Capps et al., 2012).

Le développement professionnel diffère selon le moment de la carrière des enseignants. Ces derniers ont davantage recours à l'observation de leurs pairs et au mentorat au début de leur carrière et à la lecture en fin de carrière alors qu'au milieu de celle-ci, ils utilisent des moyens plus formels de formation (Richter, Kunter, Klusmann, Lüdtke et Baumert, 2011).

Ces recherches indiquent que les enseignants jouent un rôle dans leurs apprentissages professionnels et présentent le contexte dans lequel ils se produisent. Cependant, elles n'indiquent pas comment les enseignants parviennent à les réguler. L'ARA est l'avenue considérée dans cette recherche.

Certaines recherches portent surl'ARA des futurs enseignants, mais aucune ne s'intéresse spécifiquement aux enseignants en exercice ${ }^{2}$. La nature très différente de ces études ne permet pas d'établir une comparaison de résultats, mais trace plutôt le portrait de l'ARA des futurs enseignants (Dignath et Buettner, 2008; Endedijk, Vermunt, Verloop et Brekelmans, 2012; Kramarski et Michalsky, 2009).

Les apprentissages réalisés en milieu scolaire, y compris la formation initiale, se déroulent souvent en contexte formel. Comme la plupart des recherches sur l'ARA des enseignants ont porté sur les futurs enseignants, cela correspond peu au contexte d'apprentissage des enseignants en exercice. Puisque ces derniers suivent leur rythme, poursuivent leur finalité et autoévaluent leurs résultats, l'apprentissage réalisé au travail est moins intentionnel et planifié tout en étant plus contextuel et collaboratif (Endedijk et al., 2012). Les situations liées à l'enseignement se produisent rarement dans un contexte stable, où les problèmes sont bien définis et où les buts et les valeurs sont partagés par l'ensemble des enseignants (Endedijk et al., 2012). L'ARA représente donc une manière personnelle de structurer les apprentissages professionnels.

Les recommandations concernant les recherches à réaliser sur l'ARA comprennent la considération à accorder aux caractéristiques personnelles des participants. En effet, il est mentionné que l'âge peut influencer les stratégies d'autorégulation utilisées, les modalités et la durée des interventions mises en

2 - Formation et profession 29(2), 2021 
place (Dignath et Buettner, 2008). Il est également indiqué que l'ARA mérite d'être examinée à partir du profil d'études et de l'âge de l'apprenant (Kramarski et Michalsky, 2009) ou selon le moment de la carrière et l'expérience des enseignants (Capa-Aydin et al., 2009). Le choix des participants de cette recherche tient compte de ces recommandations.

\section{Cadre théorique}

\section{Autorégulation de l'apprentissage (ARA)}

La proposition théorique de l'ARA de Zimmerman et Labuhn (2012) s'appuie sur la théorie sociocognitive de l'apprentissage de Bandura (1986). L'ARA regroupe les processus activant et maintenant la cognition, les comportements et l'affect orientés vers l'atteinte d'un but d'apprentissage (Zimmerman, 2002). Cette proposition considère que l'ARA est un cycle composé de trois phases (figure 1). La planification influence le contrôle d'exécution qui, à son tour, influence l'autoréflexion. Un cycle est complété quand l'autoréflexion a un impact sur la phase de planification des apprentissages ultérieurs.

La planification implique les processus et les croyances précédant les efforts d'apprentissage et en établissant les assises. Elle est constituée de l'analyse de la tâche et des croyances motivationnelles qui, elles-mêmes, se déclinent en sous-processus (Zimmerman, 2002).

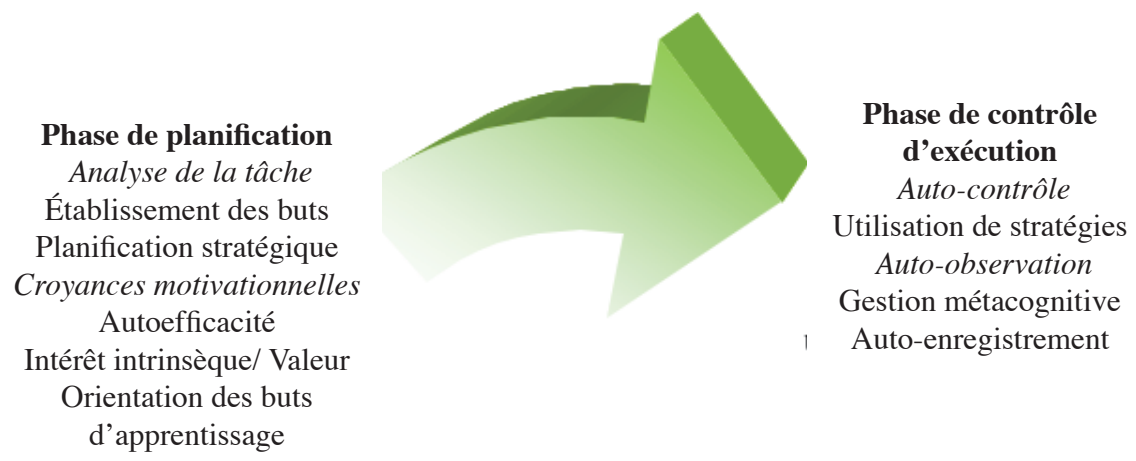

\section{Figure 1}

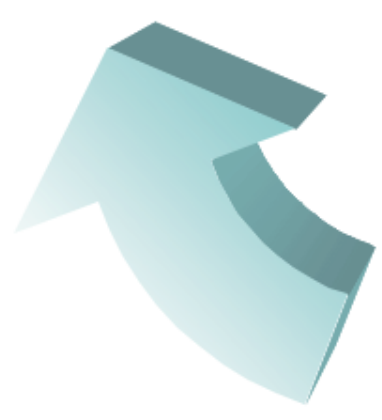

Phase d'autoréflexion

Auto-jugement

Auto-évaluation

Attribution causale

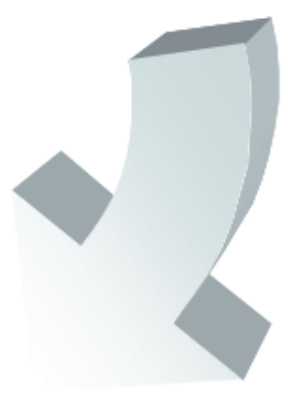

Auto-réaction

Auto-satisfaction/affectivité

Adaptativité

Phases et processus de l'ARA (d'après Zimmerman et Labubn, 2012) 
Le contrôle d'exécution regroupe les processus survenant lors des efforts d'apprentissage et affectant la concentration et la performance (Zimmerman, 2002). Il inclut l'autocontrôle et l'auto-observation qui sont également composés de sous-processus.

L'autoréflexion regroupe les processus et les croyances intervenant après les efforts d'apprentissage influençant les réactions de l'apprenant devant cette expérience d'apprentissage (Zimmerman, 2002). Cette phase inclut l'autojugement et l'autoréaction qui se subdivisent à leur tour en sous-processus.

\section{Développement professionnel}

Selon Uwamariya et Mukamurera (2005), il existe deux perspectives au développement professionnel : développementale et professionnalisante. Une perspective développementale est vue comme un phénomène de croissance progressif constitué d'étapes. Dans la problématique, il est mentionné que l'âge et le niveau d'expérience des apprenants ont une incidence sur l'ARA. Dans cette recherche, les cinq étapes de Huberman (1989) sont retenues et synthétisées en trois grands moments : début de carrière (0-6 ans), milieu de carrière (7-18 ans) et fin de carrière (19-30 ans). Ces étapes permettent de situer les participants de cette recherche.

Par ailleurs, Uwamariya et Mukamurera (2005) indiquent que, lorsqu'une perspective professionnalisante est adoptée, cela peut se faire selon deux orientations. Compte tenu des choix faits dans cette recherche, l'orientation s'intéressant aux processus d'apprentissage est retenue. À partir d'une synthèse de définitions de plusieurs auteurs (Barbier, Chaix et Demailly, 1994; Clement et Vandenberghe, 2000; Day, 1999; Donnay et Charlier, 2006; Wells, 1993), le développement professionnel est défini comme l'ensemble des processus d'apprentissage permettant la construction de compétences et de l'identité professionnelle. Le développement professionnel est continu, contextualisé et orienté vers l'atteinte d'un but, sans être entièrement planifiable, puisqu'il se déroule de manière formelle et informelle, individuellement et collectivement. Le développement professionnel des enseignants induit un apprentissage visant, entre autres, l'amélioration des pratiques (Deaudelin, Lefebvre, Brodeur, Mercier, Dussault et Richer, 2005; Guskey, 2002). Il est donc généralement admis que le développement professionnel peut avoir un impact sur la réussite des élèves par le biais d'un changement positif de ces pratiques (Guskey, 2002).

\section{Pratiques d'enseignement (PE)}

Le concept des PE est plus restreint que celui de pratiques enseignantes puisqu'il vise directement l'apprentissage des élèves. Tout comme les pratiques enseignantes, les $\mathrm{PE}$ comportent des actes singuliers et situés, observables ou non (Altet, 2002). Elles sont effectuées en présence d'élèves ou non, durant le temps scolaire et en dehors de ce dernier, individuellement ou collectivement (Deaudelin et al., 2005).

Altet (2002) définit l'enseignement comme «un processus interactif, interpersonnel, intentionnel, finalisé par l'apprentissage des élèves» (p. 85). Elle ajoute que la pratique possède une dimension comportementale comprenant les actes observables, les actions, les réactions et les procédés de mise en œuvre par une personne dans une situation donnée ainsi qu'une dimension cognitive englobant les choix et les prises de décision.

4 - Formation et profession 29(2), 2021 
Les PE comportent trois phases : préactive (actions réalisées par les enseignants lors de la planification de l'enseignement), interactive (interventions réalisées par les enseignants en présence des élèves) et postactive (actions liées au retour sur l'enseignement) (Deaudelin et al., 2005).

\section{Objectif de recherche}

Compte tenu de ces considérations, l'objectif général de la recherche est de décrire l'ARA d'enseignants en exercice au cours du développement de la compétence ciblée,à partir de l'analyse des PE liées à celle-ci. Lobjectif spécifique de cet article est de présenter les quatre intersections identifiées entre les phases des PE et de l'ARA.

\section{Méthodologie}

\section{Sélection des participants}

Cette recherche descriptive (Fortin,1996), réalisée sous la forme d'une étude de cas multiples (Yin,2009), s'est effectuée auprès de trois enseignants à des moments différents de la carrière professionnelle.

Ces cas ont été choisis à partir du principe de diversification interne (Poupart, Groulx, Deslauriers, Laperrière, Mayer et Pires, 1997). Les cinq premiers critères obligatoires assuraient l'homogénéité du groupe de référence. Les enseignants devaient être légalement qualifiés, enseigner en classe ordinaire au secondaire pour des raisons de convenance, être en poste pendant la collecte de données, être formés dans un autre domaine que celui de l'adaptation scolaire et s'engager dans le développement de la compétence ciblée.

À l'intérieur de ce groupe, le critère discriminant assurait la diversification interne (Poupart et al., 1997). Il impliquait l'appartenance à l'un des moments de la carrière professionnelle. Un seul enseignant par étape a été retenu :

a) Début de carrière : Chlorophylle ( $\mathrm{Ch})$ (6 ans d'expérience);

b) Milieu de carrière : Frédérique (Fr) (11 ans d'expérience);

c) Fin de carrière : Clément $(\mathrm{Cl})$ (37 ans d'expérience).

\section{Méthodes et outils de collecte de données}

La collecte de données s'est échelonnée sur 10 mois et comportait quatre entrevues, deux entretiens d'explicitation et deux observations réalisées en classe par la chercheure. Différents artefacts, produits au cours des phases des PE et de l'ARA, ont été recueillis. Les différents outils utilisés pour la collecte de données sont présentés selon qu'ils documentent l'apprentissage réalisé pendant les phases des PE ou celles de l'ARA. 


\section{Pratiques d'enseignement (PE)}

Pour chacune des phases de PE, les méthodes de collecte de données retenues devaient favoriser l'accès aux pratiques déclarées et aux pratiques constatées, permettre d'appréhender les phases préactive, interactive et postactive des $\mathrm{PE}$ et donner accès aux dimensions cognitives et comportementales (tableau 1). Les codes indiqués dans le tableau servent à classer les données selon leur outil de collecte. Par exemple, les données de Clément, recueillies lors de la $4^{\mathrm{e}}$ entrevue, deviennent (ClA4).

\section{Tableau 1}

Méthodes et outils de collecte de données retenues (PE)

\begin{tabular}{|c|c|c|c|}
\hline \multirow{2}{*}{$\begin{array}{c}\text { PE } \\
\text { Phase préactive }\end{array}$} & Dimension & Pratiques déclarées & Pratiques constatées \\
\hline & $\begin{array}{l}\text { Cognitive } \\
\text { Comportementale }\end{array}$ & $\begin{array}{l}\text { Entrevue semi-dirigée } \\
\text { (A1 à A4) }\end{array}$ & $\begin{array}{l}\text { Artefacts (documents } \\
\text { activités et matériel pédagogique, etc.) } \\
\text { (Z1 à Z20) }\end{array}$ \\
\hline Phase interactive & $\begin{array}{l}\text { Cognitive } \\
\text { Comportementale }\end{array}$ & $\begin{array}{l}\text { Entretien } \\
\text { d'explicitation (P1 et P2) }\end{array}$ & $\begin{array}{l}\text { Observation (01 et 02) } \\
\text { Artefacts (activités présentées en classe, } \\
\text { notes écrites au tableau, etc.) (Z1 à Z20) }\end{array}$ \\
\hline Phase postactive & $\begin{array}{l}\text { Cognitive } \\
\text { Comportementale }\end{array}$ & $\begin{array}{l}\text { Entrevue semi-dirigée } \\
\text { (A1 à A4) }\end{array}$ & $\begin{array}{l}\text { Artefacts (matériel } \\
\text { pédagogique annoté, etc.) (Z1 à Z20) }\end{array}$ \\
\hline
\end{tabular}

\section{Autorégulation de l'apprentissage (ARA)}

Pour rendre compte de l'ARA, les méthodes de collectes de données rétrospectives se sont appuyées sur la proposition théorique de Zimmerman et Labuhn (2012) (tableau 2). Les méthodes choisies étaient variées et complémentaires afin de donner accès au regard du participant et de la chercheure. 


\section{Tableau 2}

Méthodes de collecte de données retenues (ARA)

\begin{tabular}{|c|l|l|l|}
\hline $\begin{array}{c}\text { Phases de l'ARA et } \\
\text { processus }\end{array}$ & $\begin{array}{l}\text { Planification } \\
\text { Analyse de la tâche Établissement } \\
\text { des buts Planification stratégique } \\
\text { Croyances motivationnelles } \\
\text { Autoefficacité } \\
\text { Intérêt intrinsèque/valeur } \\
\text { Orientation des buts d'apprentissage }\end{array}$ & $\begin{array}{l}\text { Contrôle d'exécution } \\
\text { Autocontrôle } \\
\text { Utilisation des stratégies } \\
\text { Auto-observation } \\
\text { Gestion métacognitive } \\
\text { Auto-enregistrement }\end{array}$ & $\begin{array}{l}\text { Autoréflexion } \\
\text { Autojugement } \\
\text { Autoévaluation } \\
\text { Attribution causale } \\
\text { Autoréaction Autosatisfaction/ } \\
\text { affect Adaptativité }\end{array}$ \\
\hline Méthodes rétrospectives & $\begin{array}{l}\text { Portfolio constitué d'artefacts (notes, schémas, etc.) (Z1 à Z20) } \\
\text { Entrevue semi-dirigée (A1 à A4) }\end{array}$ \\
\hline
\end{tabular}

\section{Méthode d'analyse des données}

Dans cette recherche, cinq des six étapes de la méthode d'analyse de contenu par théorisation ancrée ont été utilisées (Paillé, 2004). L'étape de la théorisation n’a pas été atteinte puisque les résultats n’ont pas été mis à l'épreuve par l'analyse de contre-exemples. Chaque étape de la collecte des données a immédiatement été suivie d'une étape d'analyse dans un processus itératif alimentant la prochaine étape de la collecte de données (Paillé, 2004). La codification a permis de tracer un portrait d'apprenant pour chacun des participants. À partir du cadre théorique, la catégorisation a déterminé les aspects distinctifs et leurs conditions d'existence pour les PE et pour l'ARA. La mise en relation a permis de lier les catégories entre elles et de lier entre elles leurs différentes dimensions, propriétés et conditions d'existence (Paillé, 2004). Trois démarches complémentaires se sont succédé qui ont conduit à l'élaboration de trois versions différentes des cas pour chacun des participants ${ }^{3}$. La troisième et dernière démarche d'analyse a confirmé les intersections tout en limitant la redondance des résultats. Dans l'entrevue de validation, les participants ont attesté qu'ils apprennent essentiellement en fonction des besoins issus de leurs $\mathrm{PE}$, ce qui a corroboré cette dernière version. L'intégration des données a permis de représenter conceptuellement les intersections entre les PE et l'ARA. La modélisation a permis la représentation de ce qui s'applique aux trois cas simultanément en se penchant, d'abord, sur les résultats du cas se trouvant en fin de carrière, pour qui les $\mathrm{PE}$ sont, en principe mieux maîtrisé, vers la moins expérimentée. Cette façon de déconstruire l'apprentissage professionnel des enseignants et leurs $\mathrm{PE}$ a permis d'identifier des points de convergence et de divergence d'un cas à l'autre.

\section{Présentation et discussion des résultats}

L'analyse réalisée à partir des phases des PE et de l'ARA a permis l'identification de points de convergence pour quatre intersections entre les PE et l'ARA chez les trois participants : postactive et planification, préactive et contrôle d'exécution, interactive et contrôle d'exécution, postactive et autoréflexion. Dans cette section, les résultats sont présentés en même temps que la discussion pour illustrer ces intersections. 


\section{Intersection des phases - postactive (PE) et planification (ARA)}

Lorsqu'ils établissent leur but d'apprentissage, les trois participants s'appuient sur des problèmes issus de leur PE, s'attendant à ce que l'apprentissage réalisé leur soit utile et leur serve rapidement, ce que Bakkenes et al. (2010) corroborent. Capps et al. (2012) soulignent que l'une des caractéristiques soutenant l'apprentissage professionnel est la présence de situation authentique et le fait que les enseignants puissent identifier des retombées concrètes de leurs apprentissages.

Il semble que la réflexion découlant de la pratique amène les trois participants au constat qu'ils ont beaucoup de choses à apprendre en lien avec la compétence ciblée. Dans le contexte de l'enseignement aux élèves en difficulté, malgré ses nombreuses années d'expérience, Clément rappelle qu'il n'a pas été formé pour intervenir auprès d'eux $(\mathrm{ClA} 1)$. Frédérique, malgré ses formations sur les difficultés des élèves, mentionne qu'elle manque d'outils, ayant surtout été formée sur la nature des difficultés et sensibilisée à ces dernières. Elle ajoute qu'elle n'a pas de «contrôle» ni de «poigne» (FrA2). Nouvellement en poste et enseignant dans un domaine pour lequel elle n'est pas formée, Chlorophylle mentionne avoir plusieurs besoins de formation à combler simultanément (ChA1), dont celui-là.

Les trois participants établissent des buts d'apprentissage aux niveaux de complexité différents. En effet, comme ils s'appuient sur leur enseignement antérieur, les buts d'apprentissage de Clément et de Frédérique, plus expérimentés, comprennent plus de détails que celui de Chlorophylle. Frédérique souhaite apprendre sur l'enseignement explicite de la lecture en intégrant l'utilisation du tableau blanc interactif dans ses interventions (FrA1). Clément veut apprendre sur les opérations intellectuelles requises dans son cours, apprendre à travailler avec les élèves en difficulté et identifier leurs besoins afin de mettre en place des pratiques mieux adaptées (ClA1). Chlorophylle désire «trouver des façons variées d'enseigner à ses élèves afin de favoriser la répétition des concepts à maîtriser» (ChZ4). Il est possible que l'expérience professionnelle et le moment de la carrière influencent la nature du but d'apprentissage des participants. Il est également possible que le fait d'en connaître très peu sur le contenu à enseigner rende difficiles l'analyse de la tâche et l'établissement d'un but d'apprentissage spécifique. D'ailleurs, Chlorophylle mentionne devoir connaître ce qu'elle doit enseigner pour déterminer ses apprentissages : «C'est vraiment à partir du moment où je sais ce quion fait que je me mets à travailler dessus»(ChA1).

\section{Intersection des phases - préactive (PE) et contrôle d'exécution (ARA)}

Les trois participants, bien qu'ayant une démarche personnelle d'apprentissage différente, ont tendance à produire du matériel et des activités d'enseignement correspondant à leurs besoins d'apprenant (ChP1; FrP1; ClP1). Clément indique d'ailleurs qu'il s'agit de sa «zone de confort» (C1A1). Cela se manifeste, bien que les trois participants enseignent à des élèves en difficulté qu'ils sont conscients que ces élèves apprennent différemment d'eux et que leur approche ne fonctionne pas avec tous leurs élèves (ChA1; FrA1; ClA1).

La démarche d'apprentissage des trois participants diffère. En effet, Clément procède par essais-erreurs, utilisant la recherche d'informations de manière autodidacte tout en recevant peu de rétroactions extérieures ( $\mathrm{ClA} 1 ; \mathrm{ClA} 2)$. Clément a du temps pour réfléchir à ses élèves en difficulté au cours de sa planification. Il élabore des activités qu'il expérimentera, sans savoir si cela va fonctionner (ClA1). Le fait d'y penser constitue, pour lui, une démarche suffisante dans son apprentissage. Il mentionne : 
s'installer, prendre connaissance, s'approprier, étudier, comprendre, essayer des choses, "réfléchir à ça» et réajuster (ClA1; ClA3). Cette façon de faire est cohérente avec les résultats de Richter et al. (2011).

Pour sa part, Frédérique suit un "fil conducteur» et une ligne "pratique» (FrA1; FrA3). Elle envisage d'apprendre dans des formations formelles et informelles (FrA1). Cette façon de faire est compatible avec celle des enseignants en milieu de carrière (Richter et al., 2011). Frédérique apprend dans un contexte où elle peut recevoir de la rétroaction au cours des phases préactive et interactive des $\mathrm{PE}$, lui permettant de réajuster son enseignement. Certains auteurs (Dignath et Buettner, 2008) indiquent que l'expérience sociale constitue une source importante de soutien à l'ARA et augmente la capacité à résoudre des problèmes (Waitoller et Artiles, 2013). Frédérique élabore des activités d'enseignement à la suite de la formation. Elle bénéficie du soutien d'une conseillère pédagogique en dehors de la formation suivie, ce qui lui permet de poser les questions surgissant de sa pratique et elle échange avec sa collègue sur des situations vécues. Ces actions rejoignent les résultats de Capps et al. (2012). Frédérique ne privilégie pas la démarche autodidacte, car «je n’ai pas le temps pour ça» (FrP1).

Chlorophylle décortique ses apprentissages pour avoir une «vue d'ensemble» (ChA1). Elle s'imagine en train d'enseigner et fait «tout le cheminement de l'élève» (ChA4; ChP1). Pour y parvenir, elle regarde le contenu du chapitre, identifie les activités à faire, les effectue comme ses élèves, note les endroits où elle accroche, va voir sa collègue pour situer les éléments les uns par rapport aux autres et comprendre ce qu'elle ne maîtrise pas (ChA3). Ensuite, elle organise le cours qu'elle va donner. Lorsqu'elle a une question, elle la consigne, présumant que cette dernière lui sera posée par ses élèves $(\mathrm{ChP} 1)$. Chlorophylle effectue ses apprentissages et enseigne dans un contexte où elle doit préparer différents cours touchant plusieurs niveaux et de nombreuses matières. Richter et al. (2011) confirment que l'observation et le mentorat sont des approches fréquemment utilisées en début de carrière.

En contexte professionnel, l'enseignant peut apprendre en faisant ou en expérimentant (learning by doing), la plupart du temps seul, par essais-erreurs. Il peut apprendre en interaction ou en coopération avec les autres (learning in interaction), soit avec ses élèves, dans et à l'extérieur de la classe, de manière informelle avec un collègue ou en participant à des formations formelles. Il peut apprendre en lisant, en étudiant et en traitant de l'information (learning by reading). Il peut apprendre en réfléchissant (learning by thinking) à des aspects de sa pratique (Bakkenes et al., 2010; Endedijk et al., 2012). Dans cette recherche, les participants apprenaient en faisant (Clément, Frédérique, Chlorophylle), en interagissant avec les autres (Frédérique), en lisant (Clément, Frédérique et Chlorophylle) et en réfléchissant (Clément).

\section{Intersection des phases - interactive (PE) et contrôle d'exécution (ARA)}

En classe, les trois participants mentionnent valoriser la justification des réponses données et le questionnement à l'oral comme stratégies d'enseignement. Cette approche fait l'unanimité pour aller chercher les élèves en difficulté de leur classe (ChO1; FrO1; ClO1). L'analyse des données montre que cela constitue également une forme de rétroaction pour eux (ChP1; FrP1; ClP1). En effet, les commentaires et les questions des élèves leur indiquent les contenus moins bien compris et les amènent à se questionner lors de la planification du cours suivant. Cet effet de rétroaction est très présent pour Chlorophylle (ChP1). Cette dernière, ne connaissant pas les contenus à l'avance, découvre en 
enseignant les liens que les élèves font et qu'elle n'avait pas anticipés. Elle répond à des questions pour lesquelles elle n'a pas les réponses et pour lesquelles elle se fait "prendre au piège» (ChP1). À l'occasion, les élèves lui indiquent les erreurs qu'elle a faites dans son explication ou elle les constate lorsqu'un élève produit une erreur à la suite de son enseignement (ChO1). Elle ajoute que "Là, tu gères autre chose en même temps» (ChP1).

Frédérique, qui enseigne avec sa collègue, mentionne apprendre en la regardant enseigner. Elle découvre ainsi de nouvelles façons d'expliquer ou des manières différentes d'aborder le contenu en pouvant «confronter nos idées» (FrA3).

Même si l'aspect de la rétroaction semble moins présent chez Clément, la phase interactive fait ressortir ce qui lui reste à peaufiner. L'expérimentation du matériel et des activités d'enseignement créés lui permet de procéder à des ajustements après avoir enseigné : "Ce sont les attitudes des élèves qui me guident beaucoup et le feeling que je sens chez eux et dans les activités que je fais» (ClA3).

Par ailleurs, le temps est un facteur mentionné par tous les participants pendant cette phase de l'ARA. Ils soulignent avoir besoin de temps pour actualiser leurs apprentissages dans les PE. Plus l'échéancier est rapproché, plus l'apprentissage se fait près de la phase préactive et se complète au cours de la phase interactive, au moment où l'interaction avec les élèves ou les collègues met en lumière les aspects des apprentissages non maîtrisés. Encore une fois, c'est Chlorophylle qui est la plus affectée par cela. Elle mentionne fonctionner plus lentement que sa collègue, possiblement en lien avec son propre rythme (ChA4). Comme il a été mentionné précédemment, la durée et le temps sont des facteurs soutenant les apprentissages (Capps et al., 2012; Waitoller et Artiles, 2013). De plus, Frédérique et Chlorophylle font des choix selon leurs priorités personnelles, familiales et professionnelles (ChA1; FrA1). Elles n'investissent pas tout le temps souhaité dans la phase préactive et en mettent davantage lorsqu'elles enseignent. Pour Clément, le temps intervient différemment. Ainsi, il semble avoir le temps de faire les apprentissages désirés (ClA1). D'ailleurs, il souligne qu'une démarche par essais-erreurs requiert du temps pour fonctionner (ClA1). En revanche, c'est le temps qui lui reste dans la profession qui l'affecte le plus. Il souligne que, «rendu à son âge» et «compte tenu du temps quỉl lui reste», cela constitue une limite pour faire de nouveaux apprentissages (C1A1; C1A2).

\section{Intersection des phases - postactive (PE) et autoréflexion (ARA)}

La phase d'autoréflexion semble plus importante pour Clément et Frédérique que pour Chlorophylle. Toutefois, Clément paraît avoir plus de difficultés à les actualiser dans sa pratique. Il mentionne qu' «il faut que je réfléchisse à la meilleure façon que je peux le donner. Des fois, il faut que je change ma façon de faire (...) pour que ça facilite ce que je veux faire avec eux autres. (...) Il faut toujours se remettre en question, toujours réévaluer ce quion fait» (ClA3). Frédérique semble parvenir plus facilement à actualiser ses réflexions dans sa pratique et mentionne que ses apprentissages lui permettent "de mieux réfléchir aux questions que l'on pose aux élèves» (FrZ14). Chlorophylle paraît surtout réagir de cours en cours : "Il a fallu que j'apprenne ça parce qu'une fois que c'est appris, c'est appris. Bon, maintenant, je vais leur montrer» (ChA3). Son autoréflexion s'oriente donc essentiellement à court terme. La recherche d'Endedijk et al. (2012) précise que les futurs enseignants procèdent de manière prospective, en planifiant, et de manière rétrospective, en s'évaluant après coup. 
Les trois participants ne parviennent pas à atteindre leur but d'apprentissage de la même façon. Clément et Frédérique, qui ont un but d'apprentissage plus complexe, indiquent n'en avoir atteint qu'une partie. Chlorophylle croit avoir atteint son but d'apprentissage, mais elle sait qu'elle ferait mieux si elle pouvait redonner le même cours ultérieurement: "Pour une première année... parce que vu la nouveauté, vu l'ampleur de la tâche de quelque chose que je ne maîtrisais pas nécessairement... oui, mission accomplie» (ChA3).

L'autoréflexion permet d'évaluer les apprentissages réalisés en s'appuyant sur les PE. Pour Clément, les réactions des élèves sont importantes dans le processus. Il parle souvent d'un jeu de balles de tennis et de baseball (ClA1; ClA2; $\mathrm{ClA} 3$; $\mathrm{ClP} 1)$, où les échanges lui indiquent si ses apprentissages, matérialisés sous la forme de matériel et d'activités d'enseignement, ont touché leurs cibles. Il constate les nouveaux apprentissages à faire en examinant les résultats des élèves, les changements dans leur attitude et leur bien-être (ClA4). Les trois participants mentionnent d'ailleurs que les résultats des élèves s'avèrent être le premier indicateur de la réussite de leurs apprentissages (ChA1; ChA4; FrA1; FrA2; FrZ11; ClA1; ClA2). Endedijk et al. (2012) confirment que les critères de réussite touchent souvent la réussite et le bien-être des élèves même si d'autres facteurs, ne relevant pas des enseignants, peuvent être impliqués. Cela s'apparente au modèle du changement chez les enseignants de Guskey (2002) où le développement professionnel entraîne des changements dans la pratique des enseignants, puis dans les résultats et les apprentissages des élèves avant d'en produire un dans les croyances et les attitudes des enseignants. Or, Chlorophylle, outre les critères s'appuyant sur les résultats des élèves, en mentionne des plus personnels. Ainsi, si elle est «plus confiante» devant la classe, si elle peut «jouer avec la matière», la donner "dans ses mots», alors elle sait qu'elle a atteint son but d'apprentissage (ChA4). Ceci n'est pas mentionné par les deux autres participants.

\section{Synthèse des intersections}

Cette discussion indique que le but d'apprentissage, présent dans la phase de planification de l'ARA, est déterminé par les problèmes de la pratique issus des phases postactives des $\mathrm{PE}$ précédant l'apprentissage choisi.

Pendant la phase préactive des PE, l'intersection avec la phase de contrôle d'exécution de l'ARA laisse entrevoir l'importance de cette phase dans l'apprentissage des enseignants. Pendant qu'ils préparent leur enseignement, ils ont la possibilité de planifier leurs apprentissages et d'apprendre pour leur enseignement.

Lors de la phase interactive des $\mathrm{PE}$, les enseignants poursuivent leurs apprentissages professionnels par le biais de la rétroaction externe et de la réflexion qu'ils font dans l'action.

Au cours de la phase postactive des PE, les enseignants évaluent leur pratique et leurs apprentissages. Cela les amène à préciser ce qu'il leur reste à maîtriser. Cette phase postactive influence donc la phase de planification du prochain apprentissage et permet de débuter de nouveaux cycles d'apprentissage. Elle contribue également à déterminer s'il y a eu des changements relatifs aux apprentissages de l'enseignant et aux résultats des élèves. 
La figure 2 présente une simplification des intersections identifiées dans cette recherche. Le cycle de l'ARA est continu et procède d'une phase de l'ARA à l'autre, les phases des PE se greffant au tout.

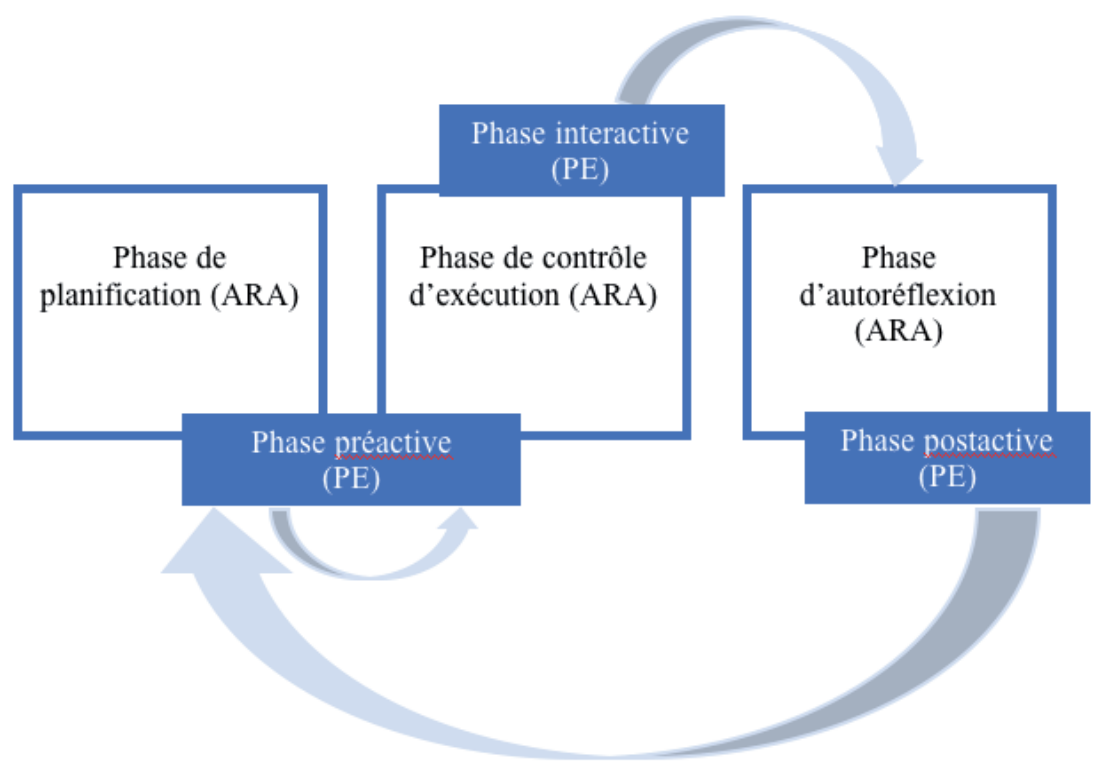

Figure 2

Intersections entre les $P E$ et l'ARA

\section{Conclusion}

Cette recherche a contribué à l'amélioration des connaissances de l'ARA d'enseignants en exercice. Les principaux résultats de l'étude ont permis de dégager quatre intersections entre les PE et l'ARA.

Sur le plan méthodologique, la nature du devis qualitatif de la recherche, reposant sur une étude de cas multiples $(n=3)$, permet une généralisation sur le plan théorique. Il n'est pas possible de brosser un portrait du développement professionnel pour chacun des moments de la carrière. Des recherches permettront d'aller plus loin dans l'hypothèse avancée. Les résultats présentés dans cet article soustendent les pistes proposées.

Premièrement, pendant la formation continue, la rétroaction externe s'appuyant sur les $\mathrm{PE}$ pourrait être utilisée puisque plusieurs apprentissages semblent alors s'actualiser.

Deuxièmement, l'apprentissage des nouveaux enseignants en exercice se déroule dans un contexte particulier. Être en mode survie, devoir faire un grand nombre d'apprentissages en amorçant une vie familiale peut ajouter un stress et teinter certaines décisions professionnelles. L'accompagnement au cours de l'insertion professionnelle semble une approche à maintenir.

Troisièmement, la recherche pourrait s'intéresser à l'apprentissage des enseignants en fin de carrière afin de définir plus précisément ses caractéristiques. La formation continue pourrait alors s'adapter à cette réalité. 


\section{Notes}

1 Dans le récent référentiel, le libellé de la compétence devient «Tenir compte de l'hétérogénéité des élèves. Mettre en place, dans le cadre d'un enseignement inclusif, des stratégies de différenciation pédagogique en vue de soutenir la pleine participation et la réussite de tous les élèves» (Gouvernement du Québec, 2020, p. 62).

2 La présentation détaillée de la recension des écrits se retrouve dans Marceau (2015).

3 Une présentation détaillée des différentes mises en relation est présentée dans Marceau (2015).

\section{Références}

Altet, M. (2002). Une démarche de recherche sur la pratique enseignante : l'analyse plurielle. Revue française de pédagogie, $138,85-93$.

Bakkenes, I., Vermunt, J. D. et Wubbels, T. (2010). Teacher learning in the context of educational innovation: Learning activities and learning outcomes of experienced teachers. Learning and Instruction, 20, 533-548.

Bandura, A. (1986). Social Foundations of Thought and Action: A Social Cognitive Theory. Englewood Cliffs, NJ: Prentice-Hall Inc.

Barbier, J. M., Chaix, M.-L. et Demailly, L. (1994). Éditorial. Recherche et formation, 17, 5-8.

Capa-Aydin, Y., Sungur, S. et Uzuntiryaki, E. (2009). Teacher self-regulation: Examining a multidimensional construct. Educational Psychology, 29(3), 345-356.

Capps, D. K., Crawford, B. A. et Constas, M. A. (2012). A review of empirical literature on inquiry professional development: Alignment with best practices and a critique of the findings. Journal of Science Teacher Education, 23(3), 291-318.

Clement, M. et Vandenberghe, R. (2000). Teachers' professional development: A solitary or collegial (ad)venture? Teaching and Teacher Education, 16(1), 81-101.

Day, C. (1999). Developing Teachers: The Challenge of Lifelong Learning. Londres : Falmer Press.

Deaudelin, C., Lefebvre, S., Brodeur, M., Mercier, J., Dussault, M. et Richer, J. (2005). Évolution des pratiques et des conceptions de l'enseignement, de l'apprentissage et des TIC chez des enseignants du primaire en contexte de développement professionnel. Revue des sciences de l'éducation, 31(1), 79-110.

Dignath, C. et Buettner, G. (2008). Components of fostering self-regulated learning among students. A meta-analysis on intervention studies at primary and secondary school level. Metacognition Learning, 3, 231-264.

Donnay, J. et Charlier, E. (2006). Apprendre par l'analyse de pratiques. Initiation au compagnonnage réflexif. : Éditions du CRP.

Endedijk, M., Vermunt, J., Verloop, N. et Brekelmans, M. (2012). The nature of student teachers' regulation of learning in teacher education. British Journal of Educational Psychology, 82, 469-491.

Fortin, M.-F. (1996). Le processus de la recherche : de la conception à la réalisation. Montréal, Québec : Décarie.

FSE - Fédération des syndicats de l'enseignement (2014). EHDAA-les positions de la FSE. http://fse.qc.net/ grandsdossiers/ehdaa/.

Gaudreau, N., Royer, É., Frenette, É., Beaumont, C. et Flanagan, T. (2013). Classroom behaviour management: The effects of in-service training on elementary teachers' self-efficacy beliefs. McGill Journal of Education/Revue des sciences de l'éducation de McGill, 48(2), p. 359-382.

Gouvernement du Québec (1999). Une école adaptée à tous ses élèves. Politique de l'adaptation scolaire. Québec : ministère de l'Éducation du Québec.

Gouvernement du Québec (2001). La formation à l'enseignement. Les orientations. Les compétences professionnelles. Québec : ministère de l'Éducation du Québec.

Gouvernement du Québec (2008). Des conditions pour mieux réussir - Plan d’action pour soutenir la réussite des élèves bandicapés ou en difficulté d'adaptation ou d'apprentissage (EHDAA). Québec : ministère de l'Éducation, du Loisir et du Sport.

Gouvernement du Québec (2010). Rencontres des partenaires en éducation. Document d'appui à la réflexion. Rencontre sur l'intégration des élèves handicapés ou en difficulté. Québec : ministère de l'Éducation, du Loisir et du Sport. 
Gouvernement du Québec (2020). Référentiel de compétences professionnelles. Profession enseignante. Québec : ministère de l'Éducation du Québec.

Goyette, N. et Martineau, S. (2018). Les défis de la formation initiale des enseignants et le développement d'une identité professionnelle favorisant le bien-être. Phronesis, 7(4), p. 4-19.

Guskey, T. R. (2002). Professional development and teacher change. Teachers and Teaching: Theory and Practice, 8(3/4), 381-391.

Huberman, M. (1989). Les phases de la carrière enseignante : un essai de description et de prévision. Rerue française de pédagogie, 80, 5-16.

Kramarski, B. et Michalsky, T. (2009). Investigating pre-service teachers' professional growth in self-regulated learning environments. Journal of Educational Psychology, 101(1), 161-175.

Lenoir, Y. (2010). La réforme de la formation à l’enseignement au Québec. Revue internationale d'éducation de Sèvres, 55, 37-48.

Marceau, N. (2015). L'autorégulation de l'apprentissage professionnel d'enseignants en exercice à l'ordre secondaire et le développement de la compétence professionnelle visant l'adaptation de leurs pratiques d'enseignement aux élèves en difficulté. (Thèse de doctorat inédite). Université de Sherbrooke, Québec, Canada.

Paillé, P. (2004). Qualitative par théorisation (méthode d'analyse de contenu). Dans A. Mucchielli (dir.) Dictionnaire des méthodes qualitatives en sciences humaines (p. 214-220), 2e édition. Paris : Armand-Colin.

Poupart, J., Groulx, L.-H., Deslauriers, J.-P., Laperrière, A., Mayer, R. et Pires, A. P. (1997). La recherche qualitative : enjeux épistémologiques et méthodologiques. Montréal, Québec : Gaëtan Morin éditeur.

Richter, D., Kunter, M., Klusmann, U., Lüdtke, O. et Baumert, J. (2011). Professional development across the teaching career: Teachers' uptake of formal and informal learning opportunities. Teaching and Teacher Education, 27(1), 116-126.

Uwamariya, A. et Mukamurera, J. (2005). Le concept de « développement professionnel» en enseignement : approches théoriques. Revue des sciences de l'éducation, 31(1), 133-155.

Waitoller, F. R. et Artiles, A. J. (2013). A decade of professional development research for inclusive education: A critical review and notes for a research program. Review of Educational Research, 83(3), 319-356.

Wells, G. (1993). Working with Teacher in the Zone of Proximal Development: Action Research on the Learning and Teaching of Science. Ontario Institute for Studies in Education.

Yin, R. K. (1984/2009). Case Study Research: Design and Methods, 4e edition. Thousand Oaks, CA: Sage Publications.

Zimmerman, B. J. (2002). Becoming a self-regulated learner: An overview. Theory into Practice, 4(2), 64-70.

Zimmerman, B. J. et Labuhn, S. A. (2012). Self-regulation of learning: Process approaches to personal development. Dans K. R. Harris, S. Graham et T. Urdan (Dir.), APA Educational Psychology Handbook : Vol. 1. Theories, Constructs, and Critical Issues (p. 399-425). Washington, D.C. : American Psychological Association.

\section{Pour citer cet article}

Marceau, N. (2021). Autorégulation de l'apprentissage professionnel et pratiques d'enseignement : intersections à considérer pour le développement professionnel des enseignants. Formation et profession, 29(2), 1-14. http://dx.doi.org/10.18162/fp.2021.560 